ARTICLE

\title{
RNF168-mediated localization of BARD1 recruits the BRCA1-PALB2 complex to DNA damage
}

\author{
John J. Krais (1) 1,3, Yifan Wang ${ }^{1,3}$, Pooja Patel ${ }^{1}$, Jayati Basu², Andrea J. Bernhardy ${ }^{1} \&$ Neil Johnson (D) ${ }^{1 凶}$
}

DNA damage prompts a diverse range of alterations to the chromatin landscape. The RNF168 E3 ubiquitin ligase catalyzes the mono-ubiquitination of histone H2A at lysine (K)13/15 ( $\mathrm{mUb}-\mathrm{H} 2 \mathrm{~A})$, forming a binding module for DNA repair proteins. BRCA1 promotes homologous recombination (HR), in part, through its interaction with PALB2, and the formation of a larger BRCA1-PALB2-BRCA2-RAD51 (BRCA1-P) complex. The mechanism by which BRCA1-P is recruited to chromatin surrounding DNA breaks is unclear. In this study, we reveal that an RNF168-governed signaling pathway is responsible for localizing the BRCA1-P complex to DNA damage. Using mice harboring a Brcal ${ }^{C C}$ (coiled coil) mutation that blocks the BrcalPalb2 interaction, we uncovered an epistatic relationship between $\mathrm{Rnf}_{168^{-}}$and $\mathrm{Brca} \mathrm{CC}^{\mathrm{Cll}}$ ales, which disrupted development, and reduced the efficiency of Palb2-Rad51 localization. Mechanistically, we show that RNF168-generated mUb-H2A recruits BARD1 through a BRCT domain ubiquitin-dependent recruitment motif (BUDR). Subsequently, BARD1-BRCA1 accumulate PALB2-RAD51 at DNA breaks via the CC domain-mediated BRCA1-PALB2 interaction. Together, these findings establish a series of molecular interactions that connect the DNA damage signaling and HR repair machinery.

\footnotetext{
${ }^{1}$ Molecular Therapeutics Program, Fox Chase Cancer Center, Philadelphia, PA, USA. ${ }^{2}$ Blood Cell Development and Function Program, Fox Chase Cancer

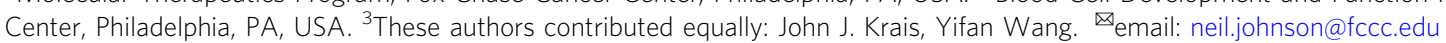


B $\mathrm{RCAl}^{\prime}$ s role in maintaining genome integrity requires numerous protein interactions. The BRCA1 really interesting new gene (RING), coiled-coil (CC), and BRCA1 C-terminal (BRCT) domains each perform specialized functions within homologous recombination (HR) DNA repair. Significantly, germline mutations found in patients with breast and ovarian cancer often cause disruption to these domains ${ }^{1,2}$.

BRCA1 and BARD1 form a heterodimer with E3 ubiquitin ligase activity via their respective RING domains. BRCA1 and PALB2 each contain CC regions that directly interact with one another, permitting the formation of a larger BRCA1-PALB2BRCA2-RAD51 (BRCA1-P) macro-complex that promotes the accumulation of RAD51 at sites of DNA damage, and consequently RAD51 filament formation ${ }^{3-5}$. While the BRCA1-PALB2 interaction is not necessary for the focal accumulation of BRCA1, it is required for efficient PALB2 and RAD51 foci formation, indicating that BRCA1 recruits PALB2-RAD51 to doublestranded DNA break (DSB) sites. The BRCA1 BRCT domainmediated interaction with ABRAXAS and RAP80 allows for the BRCA1 CC domain to simultaneously bind PALB2. Thus, it has been proposed that the BRCA1-ABRAXAS-RAP80 (BRCA1-A) complex is responsible for localizing PALB2 to DSBs ${ }^{6}$. However, several reports have demonstrated that the BRCA1-RAP80 association reduces cellular $\mathrm{HR}$ activity ${ }^{7,8}$. Moreover, RAP80 depletion was shown to have little effect on RAD51 foci formation ${ }^{7}$, making the role of RAP80-ABRAXAS in recruiting BRCA1-P ambiguous. Currently, the precise molecular mechanism responsible for the localization of the BRCA1-P complex has not been defined.

In response to DSBs, the E3 ubiquitin ligase RNF168 monoubiquitinates $\mathrm{H} 2 \mathrm{~A}$ at $\mathrm{K} 13 / 15$ (mUb-H2A), forming a binding module for several proteins, including 53BP19-11. RNF8 and RNF168 can also generate K63-linked ubiquitin polymers that promote the focal accumulation of the BRCA1-A complex ${ }^{12-14}$. In this study, we aimed to further decipher the role of RNF168 in recruiting BRCA1-P to DSBs (Fig. 1a).

\section{Results}

Rnf168 promotes Brcal-P accumulation at DSBs. We first examined phenotypes associated with mice harboring $\mathrm{Brcal}{ }^{\mathrm{CC}}$ and $R n f 168^{-}$alleles. The $B r c a 1^{C C}$ mutation specifically disrupts the Brca1-Palb2 interface ${ }^{15}$, and homozygous mice are born at reduced rates, show defective Rad51 loading and demonstrate developmental defects analogous to Fanconi anemia (FA). Rnf $168^{-1-}$ mice and cells displayed expected phenotypes (Supplementary Fig. 1a-c), including defective 53bp1 foci formation and increased Rpa32 foci positive cells (Supplementary Fig. 1d). Intercrossing $B r c a 1^{+/ C C}, R n f 168^{+/-}$mice revealed that the BrCa1 CC/CC; Rnf168-/- genotype confers early embryonic lethality (Fig. 1b and Supplementary Fig. 1e). Moreover, BrCal $1^{+/+}$; Rnf168 $8^{-1-}$ and $B r C a 1^{+/ C C}$; Rnf168 $8^{+/+}$were found at the expected rates, but $B r c a 1^{+/ C C}, R n f 168^{-/-}$mice were born at significantly reduced frequencies (Fig. 1b). Remarkably, Brca1 ${ }^{+/ C C}$; Rnf168 $8^{-/-}$ mice had traits that were similar to those observed in $B r C a 1^{C C / C C}$; Rnf168 ${ }^{+/+}$mice ${ }^{15}$, such as short stature and reduced weights (Fig. 1c), kinked tails, and areas of hypopigmented fur (Supplementary Fig. 1f). Brca1 ${ }^{+/ C C}$; Rnf168 $8^{-/-}$mice had reduced life spans (Fig. 1d), due to the onset of metastatic lymphoma (Supplementary Fig. 1g, h). Additionally, assessment of PARPi sensitivity, which is indicative of HR-deficiency, showed that Rnf168 knockout (KO) resulted in a 2.6- and 12.4-fold decrease in rucaparib LC50 values in $\mathrm{BrCa1}^{+/+}$and $\mathrm{Brca1}^{+/ C C} \mathrm{MEFs}$, respectively. However, Rnf168 KO did not affect $B r c a 1^{C C / C C}$ MEFs, which are highly sensitive from the outset (Fig. 1e and Supplementary Fig. 2a). These results are in keeping with previous work showing that Rnf168 supports HR and development in mice with alleles that reduce the Brca1-Palb2 interaction $^{16}$.

To determine whether mouse phenotypes were related to Brca1-P complex recruitment, we investigated the effects of Rnf168 KO and the Brca1 CC mutation on Brca1, Palb2, and Rad51 foci formation. As expected, Rnf168 KO resulted in loss of 53bp1 IRIF positive cells (Supplementary Fig. 2a). Of note, Brca ${ }^{C C / C C}$ cells produce a Brca1-CC protein that is stable and forms foci ${ }^{15}$. Strikingly, Brcal-WT and Brcal-CC IRIF were dramatically reduced in $R n f 168$ in $\mathrm{KO}$ cells (Fig. If and Supplementary Fig. 2a, b). The number of Palb2 and Rad51 IRIF positive $\mathrm{BrCal}^{+/+}$and $\mathrm{Brca1}{ }^{+/ C C}$ cells also decreased in the absence of Rnf168. However, Brcal ${ }^{C C / C C}$ cells, which have defective Brca1-Palb2 interaction, had few detectable Palb2 and Rad51 foci; therefore, Rnf168 KO had little measurable impact (Fig. 1f and Supplementary Fig. 2a, b). Overall, these results suggest a model whereby Rnf168 promotes Brcal localization, and consequently, the Brca1-dependent recruitment of Palb2Rad51 to DSBs.

RNF168-generated and K63-linked ubiquitin polymers are known to recruit RAP80 ${ }^{11-13}$, and thereby the BRCA1-A complex to DSB sites. To determine if RAP80 is the mediator of BRCA1-P complex recruitment, we directly compared Rap80 and Rnf168 KO MEFs for Brcal and Rad51 IRIF (Supplementary Fig. 2c). In contrast to $R n f 168^{-1-}$ cells, there was a mild reduction in Brcal, and no effect on Rad51 foci positive Rap80-/- cells (Supplementary Fig. 2c), suggesting that RNF168 recruits the BRCA1-P complex through another pathway.

RNF168-mediated localization is BRCA1 RING-dependent. BRCA1 DSB localization has previously been associated with both the RING and BRCT domains ${ }^{12-14,17-19}$. To explore mechanisms by which RNF168 promotes the recruitment of BRCA1 to DSBs, we assessed ectopic BRCA1 full-length (FL), BRCA1- $\triangle$ RING, and BRCA1- $\triangle$ BRCT foci formation (Fig. 2a). Cells expressing BRCA1$\triangle \mathrm{BRCT}$ had a moderate decrease, but BRCA1- $\triangle \mathrm{RING}$ expressing cells had significantly fewer foci positive cells and foci per nucleus, compared with BRCA1-FL expressing cells (Fig. 2b). Interestingly, BRCA1- $\triangle$ RING also demonstrated a reduction in the overall size of foci (Fig. 2b). To examine whether protein expression impacted foci formation, we compared the BRCA1- $\mathrm{RING}$ construct used above (aa 128-1863) to a more truncated construct that we previously found had improved expression and is produced from the endogenous BRCA $1^{185 d e l A G}$ locus (aa 297-1863) ${ }^{20}$. Here, foci were similar between deletions, despite BRCA1-297-1863 showing higher expression (Supplementary Fig. 2d).

We next measured the effects of RNF168 expression levels on BRCA1 foci formation. As expected, RNF168 siRNA reduced the number of foci positive cells as well as size of BRCA1-FL foci. Additionally, RNF168 siRNA resulted in a complete loss of detectable BRCA1- $\triangle$ BRCT foci; by contrast, BRCA1- $\triangle$ RING foci were unaffected (Fig. 2c). Thus, RNF168-induced BRCA1 focal accumulation requires the RING domain.

BARD1 ANK and BRCT domains promote BRCA1 foci. We aimed to decipher the mechanism by which the BRCA1 RING domain promotes foci formation. BRCA1 and BARD1 both contain N-terminal RING domains that directly interact and form an E3 ubiquitin ligase ${ }^{21}$. Because BRCA1- $\triangle$ RING does not interact with BARD1 nor engender ubiquitin ligase activity, we separately disrupted the E3 ligase activity versus the BRCA1BARD1 interaction. The BRCA1-I26A mutation is commonly used to block ubiquitin ligase activity, but may retain residual activity. However, a triple $\mathrm{I} 26 \mathrm{~A}+\mathrm{L} 63 \mathrm{~A}+\mathrm{K} 65 \mathrm{~A}$ mutation 

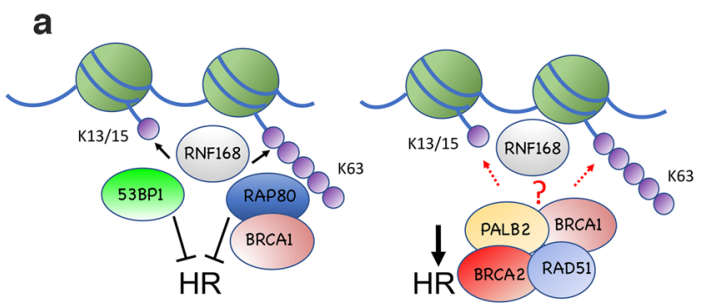

Brca1 $+/++/ \mathrm{CC} \quad+/++/ \mathrm{CC}$
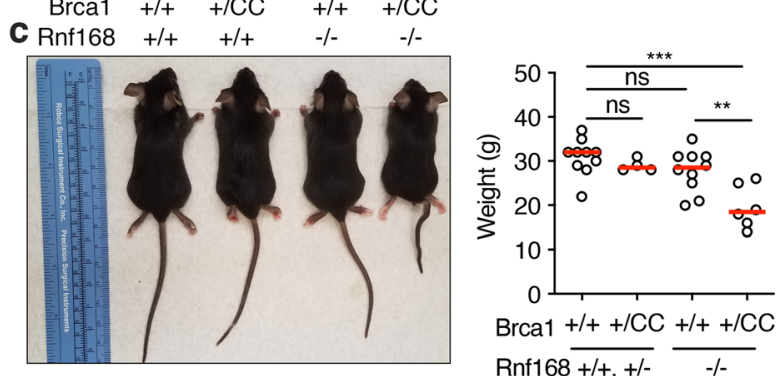

b

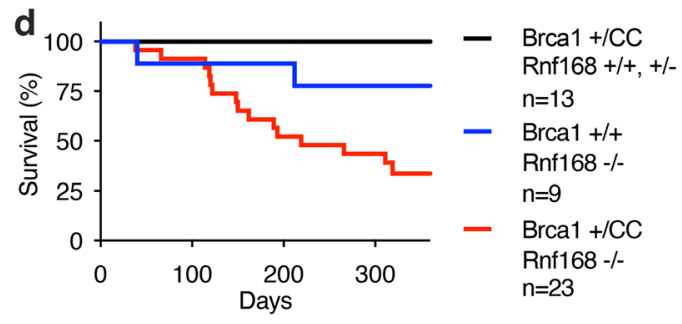

\begin{tabular}{|c|c|c|c|c|}
\hline Brca1 & Rnf168 & Expected & Observed & P value \\
\hline$+/+$ & $+/+,+/-$ & 41.06 & 66 & $<0.0001$ \\
$+/ C C$ & $+/+,+/-$ & 82.13 & 121 & $<0.0001$ \\
CC/CC & $+/+,+/-$ & 41.06 & 6 & $<0.0001$ \\
$+/+$ & $-/-$ & 13.69 & 16 & 0.5186 \\
$+/ C C$ & $-/-$ & 27.38 & 10 & 0.0004 \\
CC/CC & $-/-$ & 13.69 & 0 & 0.0001 \\
\hline \multicolumn{5}{|l}{ Total live pups: } \\
\hline
\end{tabular}

Brca1

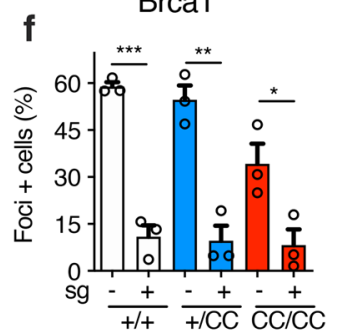

Rad51

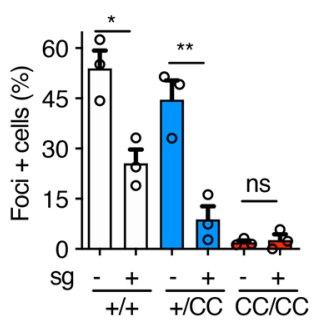

Fig. 1 Rnf168 and Brca1 mutations block development and Brca1-P localization. a Cartoon showing RNF168-mediated and H2A-associated ubiquitin modifications, as well as the influence of 53BP1, BRCA1-A (ABRAXAS-RAP80) and -P (PALB2-BRCA2-RAD51) complexes on HR. b Brca1+/CC, Rnf168 ${ }^{+/-}$ mice were intercrossed and the numbers of expected and observed live births are shown. $P$ values are obtained from two-sided chi-square goodness of fit tests for the binomial of each genotype. c Left; representative photograph. Right; weights of individual 4-6-month-old mice with the indicated genotypes,

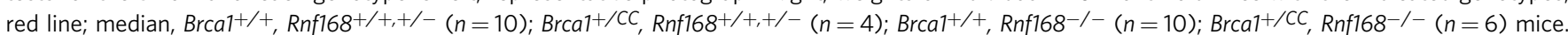
d Kaplan-Meier survival analysis of mice born with the indicated genotypes. e Brca1 ${ }^{+/+}$, Brca1 ${ }^{+/ C C}$, and Brca1CC/CC MEFs treated with GFP (-) or Rnf168 $(+)$ targeting sgRNA were subsequently incubated with increasing concentrations of the PARPi rucaparib and colony formation assessed. Mean and S.E.M. rucaparib IC50 values are shown for $n=4$ biological replicates. $\mathbf{f}$ Cells from (e) were subject to 10 Gy $\gamma$-irradiation (IR) and foci formation measured by immunofluorescence. Endogenous Brca1 and Rad51 foci, and ectopic HA-PALB2 foci formation was assessed using cell lines expressing HA-PALB2. Foci positive cells were determined to be $\geq 10 \mathrm{Brca} 1$ and Palb2 foci/nuclei, or $\geq 5$ Rad51 foci/nuclei. The mean and S.E.M. percentage foci positive cells are shown for $n=3$ biological replicates. See Supplementary Fig. 2a for representative images of cells. ${ }^{\star \star \star} p<0.001,{ }^{\star \star} p<0.01,{ }^{\star} p<0.05$, ns not significant (unpaired, two-tailed $t$-tests).

(BRCA1-3A) fully abrogates E3 ligase activity, while retaining the ability to interact with BARD122. The BRCA1-C61G protein retains the RING domain but prevents BRCA1-BARD1 heterodimerization and results in decreased protein stability ${ }^{23}$. However, we obtained cells with suitable ectopic protein expression (Fig. 3a and Supplementary Fig. 3a). We found that BRCA1 foci formation was identical between BRCA1-FL and BRCA1-3A expressing cells. By contrast, BRCA1-C61G formed fewer foci that were smaller and similar in size to the BRCA1- $\triangle$ RING protein (Fig. 3b). Similar to BRCA1- $\triangle$ RING, RNF168 siRNA also had no effect on BRCA1-C61G foci (Supplementary Fig. 3b). To further ensure that E3 ligase activity was absent, we generated a construct that physically linked BRCA1- $\triangle$ RING and BARD1$\Delta$ RING peptides (BRCA1- $\triangle$ RING-L-BARD1- $\Delta$ RING), thereby creating a synthetic heterodimer where both proteins were devoid of RING domains (Fig. 3a and Supplementary Fig. 3a). Here, BRCA1- $\triangle$ RING-L-BARD1- $\triangle$ RING IRIF was identical to BRCA1FL (Fig. 3b), suggesting that the interaction with BARD1 is required for efficient BRCA1 focal accumulation.

Interestingly, while BRCA1-FL, BRCA1-3A, and BRCA1$\triangle$ RING-L-BARD1- $\triangle$ RING expressing cells showed increased
RAD51 IRIF, BRCA1-C61G and BRCA1- $\triangle$ RING expressing cells had significantly lower levels of RAD51 IRIF (Fig. $3 \mathrm{c}$ and Supplementary Fig. 3c). PARPi sensitivity is reflective of cellular HR proficiency, and using standard colony assays, BRCA1-FL, BRCA1-3A, and BRCA1- $\triangle$ RING-L- BARD1- $\triangle$ RING expressing cells were resistant, but BRCA1-C61G and BRCA1- $\triangle$ RING expressing cells PARPi sensitive (Fig. 3d). However, we confirmed previous results using PARPi resistance colony assays ${ }^{20}$, with BRCA1-C61G and BRCA1- $\triangle$ RING showing hypomorphic activity and providing residual PARPi resistance relative to BFPexpressing control cells (Supplementary Fig. 3d).

The above results indicate that BARD1 recruits BRCA1, and consequently RAD51 to DNA damage sites. In line with the known requirement of BARD1 in providing BRCA1 protein stability $^{24}$, CRISPR/Cas9-mediated $\mathrm{KO}$ of BARD1 reduced endogenous BRCA1 protein expression levels (Supplementary Fig. 3e), as well as the number of BRCA1 and RAD51 foci in MCF7 cells (Supplementary Fig. 3f). To identify regions of BARD1 critical for BRCA1 recruitment, we expressed ectopic BARD1-FL, BARD1- $\triangle$ RING, BARD1- $\triangle \mathrm{ANK}$, and BARD1$\triangle \mathrm{BRCT}$ truncated proteins, as well as an E3 ligase disrupting 

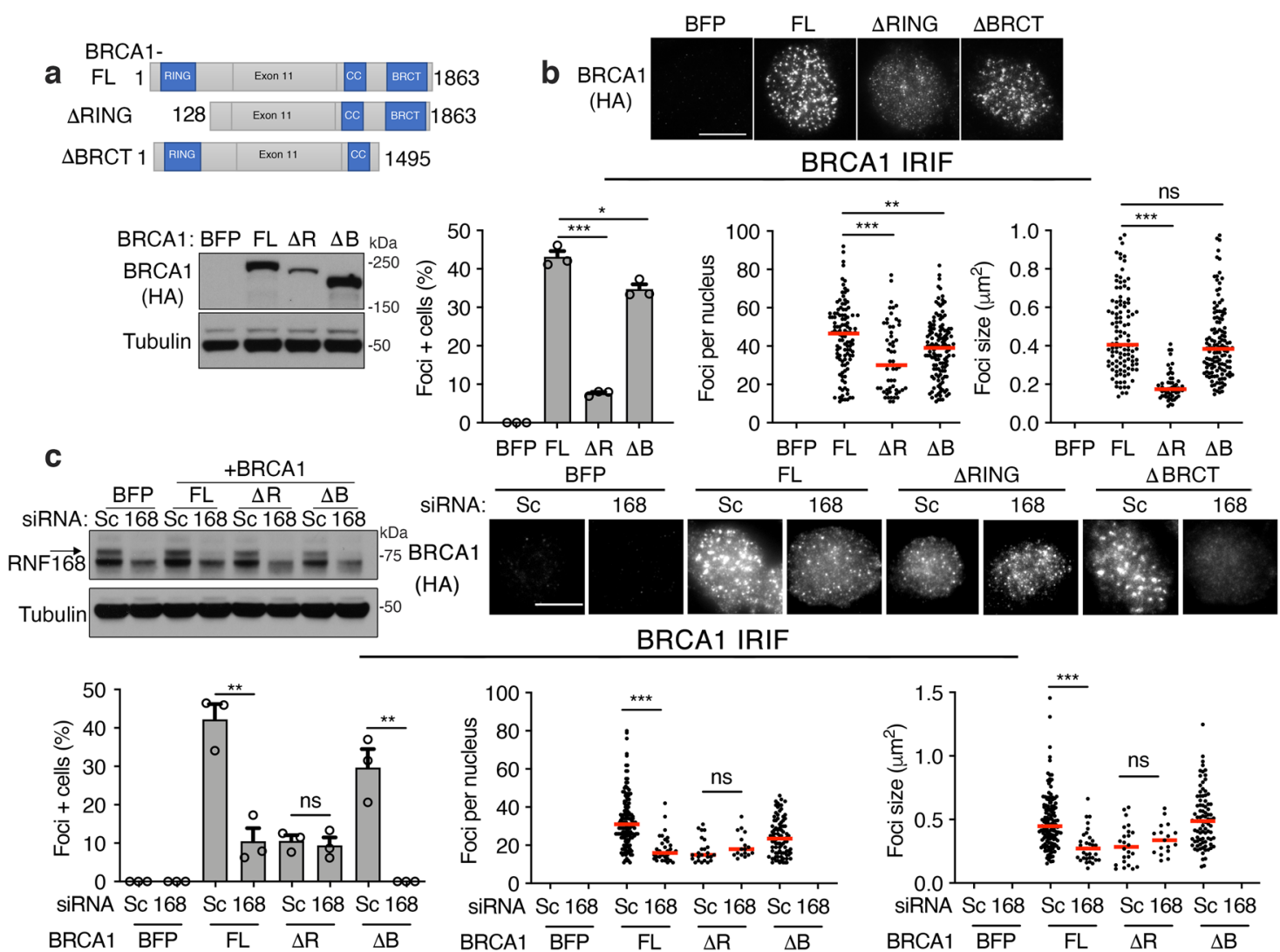

Fig. 2 RNF168 regulates BRCA1 RING-mediated foci. a Above; cartoon showing BRCA1 protein domains and truncations with aa numbers indicated. Below; BRCA1 null MDA-MB-436 cells expressing BFP control, HA-BRCA1-full-length (FL), HA-BRCA1- $\triangle R I N G(\triangle R)$, and HA-BRCA1- $\triangle B R C T(\triangle B)$ were assessed for $\mathrm{HA}$ protein levels by western blotting. $\mathbf{b}$ Cells from (a) were assessed for HA foci $8 \mathrm{~h}$ post IR by immunofluorescence. Above; representative images (scale bar, $10 \mu \mathrm{m}$ ). Left; mean and S.E.M. percentage foci positive cells; middle, foci positive cells were assessed for the number of foci present in a single nucleus, red line: median value; right, foci positive cells were assessed for the mean size of foci present per nuclei, $n=3$ biological replicates. Foci positive cells were determined to be those with $\geq 10$ foci/nuclei. ${ }^{\star \star \star} p<0.001,{ }^{\star \star} p<0.01,{ }^{\star} p<0.05$, ns not significant compared to FL (percentage positive cells: unpaired, two-tailed $t$-tests size; number and size per nucleus: nonparametric Mann-Whitney tests). See Source data for additional information. c Cells from (a) were subject to scrambled (Sc) and RNF168-targeting siRNA followed by IR and HA foci formation measured by immunofluorescence. Left; western blot showing RNF168 depletion. Above; representative cells (scale bar, $10 \mu \mathrm{m}$ ). Below; foci analyses as described in (b), $n=3$ biological replicates. See Source data for additional information.

BARD1-R99E mutation ${ }^{25}$ (Fig. 3e and Supplementary Fig. 3g). Of note, all ectopic BARD1 mutations except for BARD1- $\triangle$ RING restored endogenous BRCA1 protein expression (Fig. 3e). Using pre-extraction methods, BARD1-FL and BARD1-R99E formed similar, and BARD1- $\triangle$ RING less efficient, foci. However, BARD1- $\triangle A N K$, and BARD1- $\triangle \mathrm{BRCT}$ foci formation was barely detectable (Fig. 3f). The number of BRCA1 foci per nucleus and size of foci were markedly reduced in BARD1 KO cells, but were restored in BARD1-FL and BARD1-R99E expressing cells. In contrast, there remained fewer and smaller BRCA1 foci in BARD1- $\triangle$ RING, BARD1- $\triangle$ ANK and BARD1- $\triangle$ BRCT expressing cells (Fig. 3f). RPA32 and RAD51 foci formation followed similar patterns as BRCA1 in ectopic add back cells (Fig. $3 \mathrm{~g}$ and Supplementary Fig. 3h). PARPi resistance was also restored in BARD1-FL and BARD1-R99E, but not BARD1- $\triangle$ RING, BARD1$\triangle \mathrm{ANK}$ and BARD1- $\triangle \mathrm{BRCT}$ expressing cells (Fig. $3 \mathrm{~h}$ ). These results establish the BRCA1-BARD1 interaction, as well as the BARD1 ANK and BRCT domains, as mediators of BRCA1 focal accumulation.

RNF168-mUb-H2A-BARD1 anchors BRCA1-P. A recent study revealed that BARD1 localizes to DNA damage sites through a dual mechanism involving both the ANK and BRCT domains ${ }^{26}$. The BARD1 ANK domain is a reader of histone H4 unmethylated at $\mathrm{K} 20(\mathrm{H} 4 \mathrm{~K} 20 \mathrm{me} 0)^{17}$, and the BRCT repeat was shown to contain a BRCT domain ubiquitin-dependent recruitment motif (BUDR), which directly binds mUb-H2 ${ }^{26}$. Indeed, MCF7 $B A R D 1^{-1-}$ cells engineered to express a BARD1 ${ }^{B U D R}$ mutant construct (Supplementary Fig. 4a), had identical phenotypes to $B A R D 1^{\triangle B R C T}$ expressing cells, with inefficient BARD1 DSB localization (Fig. 4a and Supplementary Fig. 4b), and failed to rescue BRCA1 and RAD51 foci formation (Fig. 4a, b and Supplementary Fig. 4b), nor PARPi sensitivity (Fig. 4c).

Given the established role of RNF168 E3 ligase activity in generating mUb-H2 $\mathrm{A}^{10}$, we reasoned that RNF168 promotes BRCA1-P recruitment to DSB sites via BARD1 BUDR binding to mUb-H2A. To further investigate this possibility, we assessed a series of ectopic add back RNF168 mutations using an MDA-MB$231 R N F 168^{-1-}$ clone (Supplementary Fig. 4c, d). Similar to results in MEFs, BRCA1, and RAD51 IRIF were significantly reduced in MDA-MB-231 $R N F 168^{-1-}$ cells (Supplementary Fig. 4e). The RNF168 $8^{\triangle R I N G}$ mutation results in loss of the RING domain and E3 ligase activity, whereas $R N F 168^{R 57 D}$ retains E3 ligase activity but specifically blocks the ability of RNF168 to mono-ubiquitinate $\mathrm{H} 2 \mathrm{~A}^{10}$. Additionally, $R N F 168^{\triangle P I D}$ lacks the C-terminally located PALB2-interacting domain (PID), which is required for RNF168 to directly interact with PALB2 and facilitate the latter's foci formation in the absence of $\mathrm{BRCA}^{27}$ 

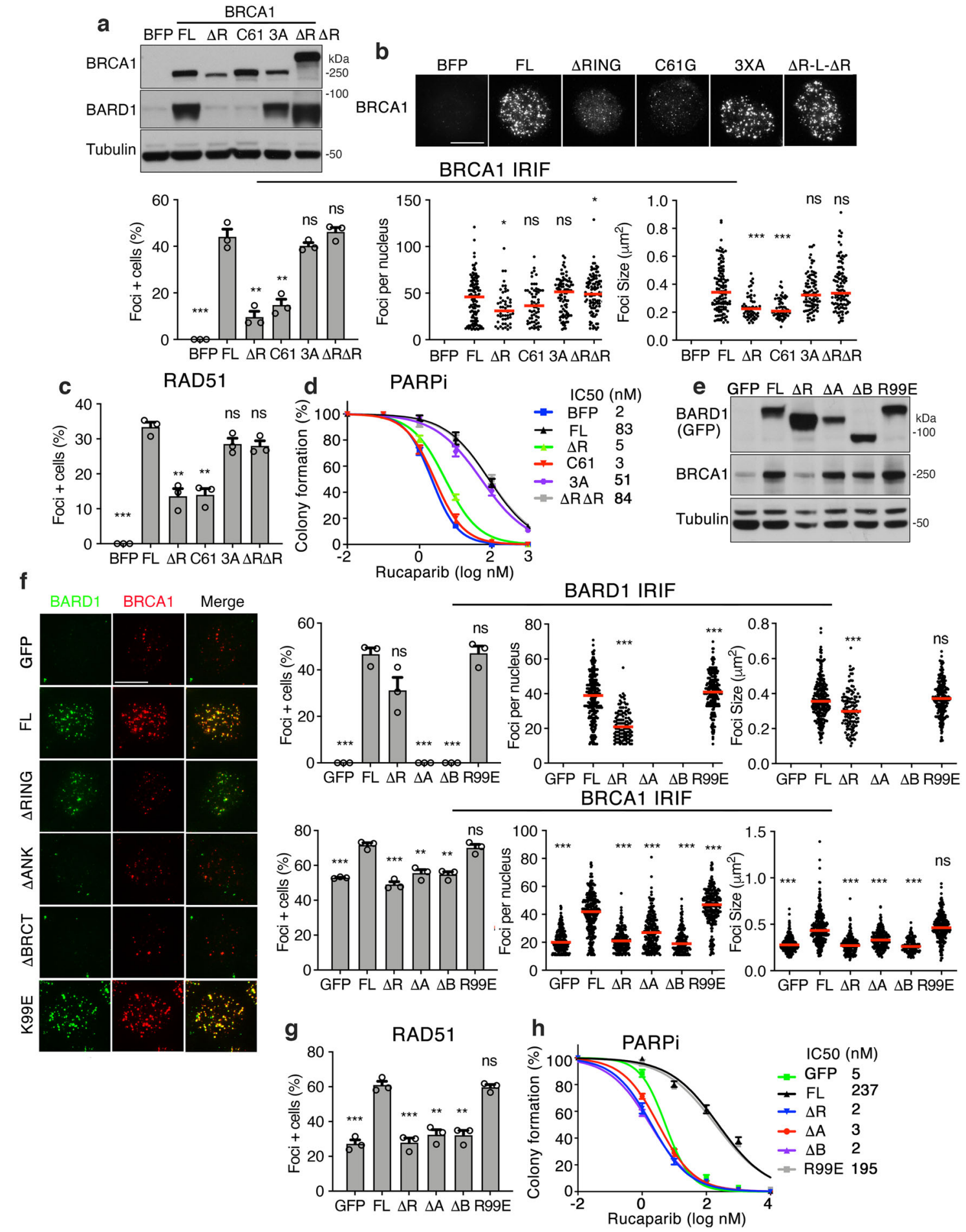

(Fig. 4d). The mRNA expression of ectopic constructs was within a similar range, but RING domain mutant protein levels were dramatically reduced compared to wild-type (Supplementary Fig. 4f), indicating that RING mutations are detrimental to protein stability. To bypass this issue, we used a $C M V$ promoter for RING mutant constructs, which produced cell lines with comparable protein expression to UBC promoter-driven $R N F 168^{W T}$ and $R N F 168^{\triangle P I D}$ expressing cells (Fig. 4d). IRIF experiments revealed that $\mathrm{RNF} 168^{\mathrm{WT}}$ and $\mathrm{RNF} 168^{\triangle \mathrm{PID}}$ formed robust foci and fully rescued 53BP1 IRIF. In contrast, RNF168 $8^{\triangle \mathrm{RING}}$ and RNF168 $8^{\mathrm{R} 5 \mathrm{D}}$ formed smaller foci and failed to rescue 53BP1 IRIF (Supplementary Fig. 4g). Moreover, while
$\mathrm{RNF} 168^{\mathrm{WT}}$ and $\mathrm{RNF} 168^{\triangle \mathrm{PID}}$ rescued, RNF168 ${ }^{\triangle \mathrm{RING}}$ and RNF168 ${ }^{\text {R57D }}$ had no effect on BARD1 and BRCA1 (Fig. 4e and Supplementary Fig. 4g), or RAD51 foci (Fig. 4f and Supplementary Fig. 4g). These data indicate that the E3 ligase activity, and specifically the ability of RNF168 to generate mUb-H2A, is required for efficient BARD1-BRCA1-RAD51 DSB localization.

We postulated that in the absence of BRCA1, a direct association of only the BRCA1 RING domain with PALB2 may direct PALB2-RAD51 to DSBs and rescue HR. Thus, we generated a construct containing the BRCA1 RING domain linked to PALB2 cDNA (HA-RING-L-PALB2) and expressed in BRCA1 null MDA-MB-436 cells. RING-containing constructs 
Fig. 3 BARD1 ANK and BRCT domains promote BRCA1 foci formation. a MDA-MB-436 cells expressing BFP, BRCA1-full-length (FL), BRCA1- $\triangle \mathrm{RING}$ $(\Delta R)$, BRCA1-C61G (C61), BRCA1-I26A+L63 A+K65A (3A), BRCA1- $\Delta$ RING-L- BARD1- $R$ RING $(\Delta R \Delta R)$ were assessed for BRCA1 and BARD1 expression by western blotting. b Cells from (a) were assessed for BRCA1 foci $8 \mathrm{~h}$ post IR by immunofluorescence. Above; representative images (scale bar, $10 \mu \mathrm{m}$ ). Left; mean and S.E.M. percentage foci positive cells; middle, foci positive cells were assessed for the number of foci present in a single nucleus, red line: median value; right, foci positive cells were assessed for the mean size of foci present per nuclei, $n=3$ biological replicates. Foci positive cells were determined to be those with $\geq 10 \mathrm{foci} /$ nuclei. ${ }^{\star \star \star} p<0.001,{ }^{* \star} p<0.01,{ }^{\star} p<0.05$, ns not significant (percentage positive cells: unpaired, two-tailed $t$-tests size; number and size per nucleus: nonparametric Mann-Whitney tests). See Source data for additional information. c Cells from (a) were assessed for RAD51 foci $8 \mathrm{~h}$ post IR by immunofluorescence. See Supplementary Fig. 3c for representative images. Mean and S.E M. percentage ( $\geq 5$ ) foci positive cells, $n=3$ biological replicates. ${ }^{\star \star *} p<0.001,{ }^{\star \star} p<0.01$, ns not significant compared to FL (unpaired, two-tailed $t$-tests). d Cells from (a) were incubated with increasing concentrations of rucaparib for 2 weeks and colony formation assessed. Mean and S.E.M. colony formation as well as mean IC50 values, $n=4$ biological replicates. e MCF7 BARD1-/- cells expressing GFP control, GFP-BARD1-full-length (FL), GFP-BARD1- $\triangle$ RING ( $\triangle R$ R), GFP-BARD1- $\triangle$ ANK $(\triangle \mathrm{A})$, GFP-BARD1- $\triangle \mathrm{BRCT}(\triangle \mathrm{B})$, and GFP-BARD1-R99E (R99E) were assessed for GFP and BRCA1 expression by western blotting. $\mathbf{f}$ Cells from (e) were assessed for BARD1 and BRCA1 foci $8 \mathrm{~h}$ post IR by immunofluorescence. Left; representative foci (scale bar, $10 \mu \mathrm{m}$ ). Right; foci analyses as described in (b). $\mathbf{g}$ Cells from (e) were assessed for RAD51 foci as described in (c). See Supplementary Fig. 3h for representative images. $\mathbf{h}$ Cells from (e) were incubated with increasing concentrations of rucaparib for 2 weeks and colony formation assessed. Mean and S.E.M. colony formation as well as mean IC50 values, $n=3$ biological replicates.

increased BARD1 protein similar to endogenous levels observed in BRCA1 wild-type MCF7 cells (Supplementary Fig. 5a). Because MDA-MB-436 cells are BRCA1 null, and end resection is necessary for efficient PALB2-RAD51 loading ${ }^{28}$, we also subject cells to GFP- or 53BP1-targeting sgRNA, which increased RPA32 IRIF (Fig. 4g and Supplementary Fig. 5a). HA-RING-LPALB2 formed detectable foci in sgGFP cells, but HA-RING-LPALB2 and RAD51 foci were dramatically increased (Fig. 4g and Supplementary Fig. 5b), and PARPi resistance was observed in sg53BP1 cells (Fig. 4h). Moreover, we confirmed that HA-RINGL-PALB2 and RAD51 foci were entirely reliant on RNF168 activity (Fig. 4i and Supplementary Fig. 5c). Thus, the BRCA1 RING domain is an RNF168-dependent DSB localization module.

RNF8-BRCA1-A provides a backup route of recruitment. Despite numerous pathogenic BRCA1 mutations being located within the BRCT repeats, loss of the BRCT domain had relatively mild effects on BRCA1 protein localization (Fig. 2b). To gain additional insight into how BRCA1 BRCT domain mutations impact BRCA1 localization and HR, we compared several truncating mutations located at varying distances from the BRCT repeat region, which spans $\sim$ aa's 1642-1856 (Fig. 5a). We confirmed previous observations ${ }^{29}$, with stop codons located close to (aa $1558^{*}$ ) or within (aa $1814^{*}$ ) the BRCT domain resulting in reduced protein expression despite similar mRNA levels (Fig. 5a), and consequently few foci forming cells (Fig. 5b and Supplementary Fig. 5d). In contrast, the BRCA1- $\triangle \mathrm{BRCT}$ protein (aa $1495^{*}$ ), which stops $\sim 147$ aa prior to the first BRCT repeat, was stable and formed relatively robust foci (Fig. 5a, b). However, BRCA1- $\triangle$ BRCT expressing cells failed to promote CtIP, RPA32, and RAD51 IRIF, relative to full-length BRCA1 expressing cells (Fig. 5b and Supplementary Fig. 5d). Hence, even when BRCA1 is stable and can localize to DSBs, in the absence of the BRCT domain, CtIP recruitment, and consequently end resection and $\mathrm{HR}$, is inefficient.

Although loss of the BRCA1 BRCT domain or RAP80 induced mild defects in BRCA1 foci (Fig. 2a and Supplementary Fig. 2c), we hypothesized that the BRCA1-A complex was likely important for residual BRCA1-P localization in the absence of the RNF168BARD1-RING recruitment axis. Interestingly, RAP80 foci were reduced but detectable in $R N F 168^{-1-}$ MDA-MB-231 cells, potentially accounting for the residual BRCA1 foci observed (Fig. 5c). In contrast, depletion of RNF8, which generates K63linked ubiquitin chains that are required for both RNF168 and RAP80 recruitment ${ }^{12-14,30}$, resulted in a severe reduction in both RAP80 foci and BRCA1 foci (Fig. 5d). Therefore, the RNF8BRCA1-A pathway appears to be partially active in the absence of
RNF168. In support of this, RAP80 depletion dramatically reduced BRCA1 and RAD51 IRIF in RNF168 ${ }^{-1-}$ cells (Fig. 5e and Supplementary Fig. 5e). Moreover, double knockout (DKO) $B A R D 1^{-1-}, A B R A X A S^{-1-}$ MCF7 cells also had severely impaired BRCA1 and RAD51 foci relative to single KO BARD1-1- cells (Fig. 5f and Supplementary Fig. 5f), confirming the supporting role of the BRCA1-A complex. Last, ABRAXAS RNAi had no effect on cells expressing BRCA1-full-length or BRCA1- $\triangle B R C T$ proteins, but completely abrogated BRCA1- $\triangle$ RING and BRCAC61G IRIF (Fig. 5g). Thus, the BRCA1-A complex is likely critical for residual HR activity in cancers with $B R C A 1$ RING domain located mutations. Though we did not assess BRCA1-B (BACH1) or BRCA1-C (CtIP) complexes, which could also have supporting roles. All in, the recruitment of the BRCA1-P complex to DSBs occurs primarily through an RNF168-mUb-H2A-BARD1BRCA1 RING axis, with RNF8-RAP80-ABRAXAS-BRCA1 BRCT signaling providing a backup route (Fig. $5 \mathrm{~h}$ ).

\section{Discussion}

In the current study, we report an RNF168-governed pathway that is responsible for the recruitment of the BRCA1-P complex to DSBs. A BUDR was recently discovered within the BARD1 BRCT domain, which directly binds $\mathrm{mUb}-\mathrm{H} 2 \mathrm{~A}^{26}$. In support of this, mutating residues within the BUDR, or specifically blocking the ability of RNF168 to generate mUb-H2A, reduced BRCA1-P complex recruitment to DSBs.

We found human and mouse cell lines to be highly dependent on RNF168 activity for efficient BRCA1, BARD1, and RAD51 foci formation. Loss of RNF168 activity has previously been shown to reduce BRCA1 foci formation $9,11,27$. However, others reported mild or little impact ${ }^{16,31}$, and we are unable to pinpoint reasons for these discrepancies, but speculate assessment of foci versus laser-stripes, or quantification methods, could contribute. RNF168 is capable of promoting BRCA1-A complex recruitment through the production of K63-linked ubiquitin polymers that form a binding module for RAP80 ${ }^{11,13}$. Moreover, RNF8 generated K63-linked ubiquitin chains ${ }^{6,32-34}$, as well as SUMObinding ${ }^{35-37}$, can contribute to RAP80 foci. In agreement, RNF168 ${ }^{-1-}$ cells had reduced, but detectable RAP80 foci. Interestingly, BRCA1-A complex disruption was highly detrimental for BRCA1-P activity in RNF168 ${ }^{-1-}$ and $B A R D 1^{-1-}$ cells, suggesting the RAP80-ABRAXAS axis confers a backup recruitment pathway (Fig. 5h). Importantly, individual disruption of the RING or BRCT recruitment pathways were insufficient to entirely abolish BRCA1 foci, shedding light on how BRCA1 mutant cancers utilize hypomorphic proteins, which often lack these 


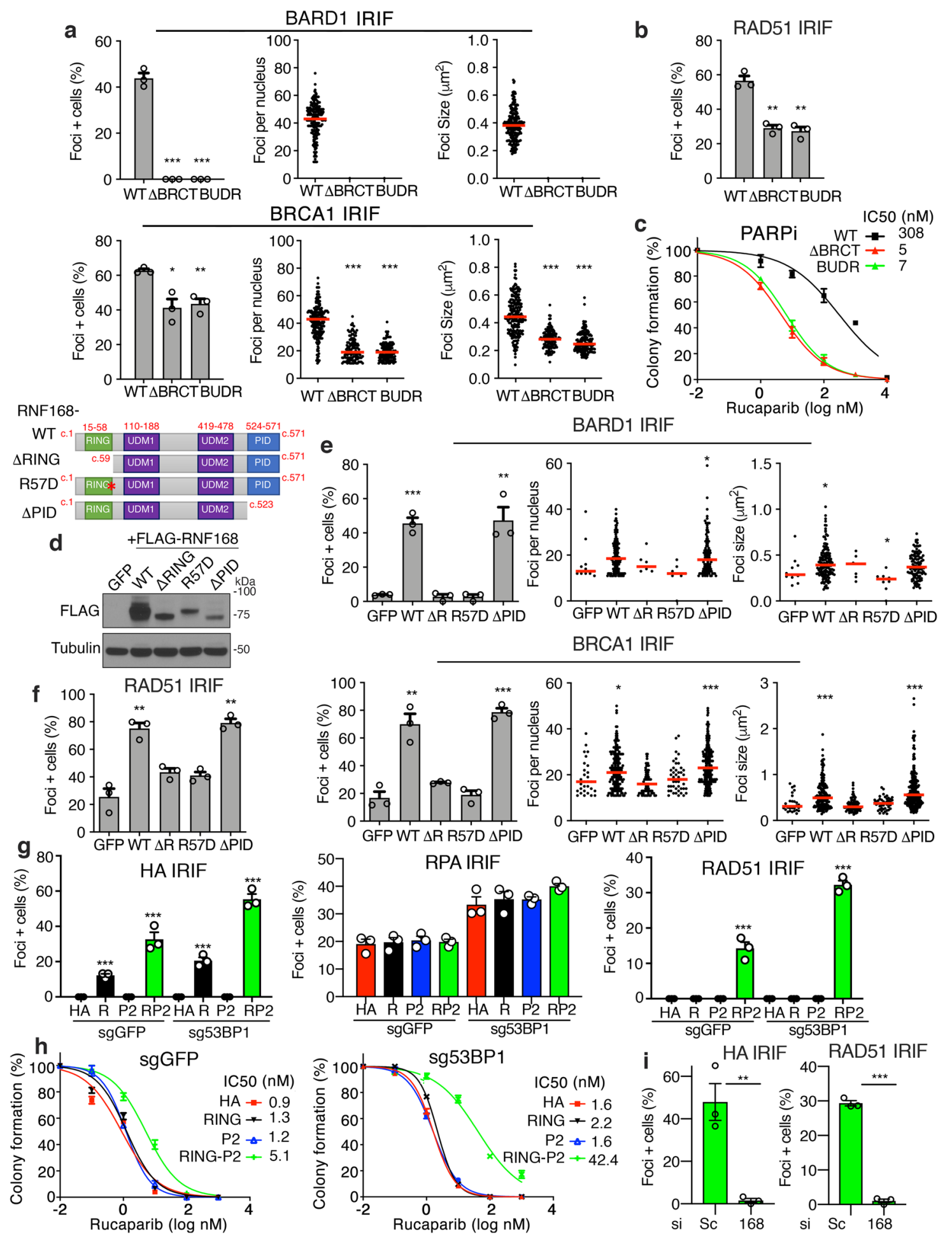

domains, to contribute to residual HR activity and PARPi resistance ${ }^{20,29,38-40}$.

We found that BRCA1 BRCT mutations often induce proteasomal degradation. Another study showed a point mutation within the BRCT domain did not affect protein expression but did reduce Brcal foci formation ${ }^{41}$; the reason for this discrepancy is unclear. Although the BRCA1-A complex provided inefficient BRCA1-P localization, it is likely sufficient to maintain residual $\mathrm{HR}$ and the relatively normal development of $\mathrm{R} n f 168^{-1-}$ mice. $B r c a 1^{+/ C C}$ reduces the number of intact Brca1-Palb2 complexes, and $B r C a 1^{+/ C C}, R n f 168^{-/-}$KO mice had a greater reduction in
Palb2 and Rad51 foci compared to either genotype alone, as well as similar developmental defects to BrcalCC/CC, Rnf168+/+ mice $^{15}$. Moreover, while we were unable to detect Palb2 and Rad51 foci in $\mathrm{Brca1}{ }^{\mathrm{CC} / C C}$ cells, it is possible that residual Rad51 loading, below the foci detection threshold, account for the birth of $\mathrm{BrCa} 1^{\mathrm{CC} / \mathrm{CC}}$ live mice. In contrast, Brcal $\mathrm{CC} / \mathrm{CC}, \mathrm{Rnf168^{-/- }}$ resulted in early embryonic lethality, potentially due to a complete abolishment of residual Rad51 loading (Supplementary Fig. 5g). Another possibility is that Rnf168 compensates for loss of Brca1 activity and directly loads Palb2 ${ }^{27}$. However, we were unable to detect Palb2 or Rad51 foci in $R n f 168^{+/+}$, Brcal ${ }^{C C / C C}$ 
Fig. 4 RNF168-mediated H2A mono-ubiquitination recruits BARD1 via the BUDR. a MCF7 BARD1-/- cells expressing GFP-BARD1-WT, GFP-BARD1$\triangle B R C T$, or GFP-BARD1-BUDR (R705A+D712A+Q715R) were assessed for BARD1 and BRCA1 foci size, number per nucleus, as well percentage foci positive cells post IR by immunofluorescence. See Supplementary Fig. $4 \mathrm{~b}$ for representative images. Left; mean and S.E.M. percentage foci positive cells; middle, foci positive cells were assessed for the number of foci present in a single nucleus, red line: median value; right, foci positive cells were assessed for the mean size of foci present per nuclei, $n=3$ biological replicates. Foci positive cells were determined to be those with $\geq 10$ foci/nuclei. ${ }^{\star \star \star} p<0.001$, ${ }^{\star \star} p<0.01,{ }^{*} p<0.05$, ns not significant compared to WT (percentage positive cells: unpaired, two-tailed $t$-tests size; number and size per nucleus: nonparametric Mann-Whitney tests). See Source data for additional information. b Cells from (a) were assessed for RAD51 foci $8 \mathrm{~h}$ post IR by immunofluorescence. Mean and S.E M. percentage ( $\geq 5)$ foci positive cells, $n=3$ biological replicates. ${ }^{\star \star} p<0.01$ compared to WT (unpaired, two-tailed $t$ tests). See Supplementary Fig. $4 \mathrm{~b}$ for representative images. c Cells from (a) were incubated with increasing concentrations of rucaparib for 2 weeks and colony formation assessed. Mean and S.E.M. colony formation as well as mean IC50 values, $n=3$ biological replicates. d Cartoon of RNF168 protein domains and mutations analyzed. Below; expression of ectopic FLAG-RNF168 proteins by western blotting in MDA-MB-231 RNF168-/- cells. e Cells from (d) were assessed for BARD1 and BRCA1 foci as described in (a) with statistical comparisons to cells expressing GFP. See Supplementary Fig. 4g for representative images. $\mathbf{f}$ Cells from (d) were assessed for RAD51 IRIF as described in (b) with statistical comparisons to cells expressing GFP. See Supplementary Fig. $4 \mathrm{~g}$ for representative images. $g$ MDA-MB-436 cells with sgRNA targeting GFP or 53BP1 were infected with virus expressing HA-RING, HA-PALB2, or HA-RING-PALB2 and assessed for HA $(\geq 10)$, RPA32 $(\geq 10)$, and RAD51 $(\geq 5)$ IRIF positive cells. Mean and S.E M. percentage foci positive cells, $n=3$ biological replicates. ${ }^{\star \star \star} p<0.001$ compared to HA alone (unpaired, two-tailed $t$-tests). See Supplementary Fig. 5a, b for Western blots and representative images. $\mathbf{h}$ Cells from $(\mathbf{g})$ were incubated with increasing concentrations of rucaparib for 2 weeks and colony formation assessed. Mean and S.E.M. colony formation, as well as mean IC50 values, are shown from $n=3$ biological replicates. i MDA-MB-436 cells with sgRNA targeting 53BP1 expressing HA-RING-PALB2 were subject to scrambled (Sc) or RNF168 siRNA and HA and RAD51 IRIF measured. Mean and S.E M. percentage foci positive cells, $n=3$ biological replicates. ${ }^{\star \star \star} p<0.001$ and ${ }^{* \star} p<0.01$ compared to Sc (unpaired, two-tailed $t$-tests). See Supplementary Fig. $5 c$ for western blots and representative images.

cells, making the role of the Rnf168-Palb2 interface unclear in this setting.

Despite both $R n f 168$ and 53bp1 KO activating resection, we speculate that the effects on Brcal mutant mouse viability differ due to the additional requirement of Rnf168 in recruiting Brcal, and subsequently Palb2-Rad51 to DSBs. Although we acknowledge that these phenotypes are not fully explained. HR and developmental defects associated with mice harboring Brcal exon 2 mutations were previously shown to be rescued by $R n f 168 \mathrm{KO}$ due to loss of 53bp1 localization and DNA end resection activation $^{16}$. Brcal exon 2 mutations produce a Brcal Ringless protein isoform, resulting in loss of Bard1 interaction ${ }^{42}$. Here, Rnf168 is dispensable for Rad51 foci and HR, possibly due to loss of the Brcal Ring domain and an inability to influence Bard1mediated Brca1-P recruitment. Thus, the BRCA1 RING domain could determine phenotypes associated with modulation of RNF168 activity in cancer. BRCA1 185delAG and C61G mutations are commonly found in patients and produce proteins that fail to interact with BARD120,23,39,43. Cancers with these mutations may reduce RNF168 activity as a means of promoting DNA end resection and inducing RAP80-mediated RINGless BRCA1-P loading, and consequently PARPi resistance. In contrast, hypomorphic proteins generated that retain the RING, may be highly dependent on RNF168 for residual BRCA1 protein activity 44 . Future studies will be required to investigate these possibilities.

BARD1 and 53BP1 both bind the same mUb-H2A modification ${ }^{26}$, implicating protein occlusion as a DSB resection regulatory mechanism. However, the switch to resection is likely more complex, given BRCA1 exon 11 and BRCT regions are also required for efficient resection ${ }^{15,16,45,46}$. Indeed, the BRCA1 RING peptide was capable of stabilizing BARD1 and localizing to DSBs, but insufficient to activate resection (Fig. 4g). Overall, we establish a series of molecular interactions linking RNF168ubiquitin signaling to BRCA1-PALB2-associated HR. Intriguingly, BRCA1 molecules may simultaneously localize to DSBs, activate resection, and promote PALB2-RAD51 loading.

\section{Methods}

Mouse models. The Fox Chase Cancer Center (FCCC) Institutional Animal Care and Use Committee (IACUC) approved experiments involving mice and all experiments were conducted in compliance with ethical regulations. Mice were housed at the FCCC Laboratory Animal Facility in standard conditions with health and pathogen monitoring by facility staff. Generation of mice harboring the
$B r c a 1^{C C}$ allele was previously described ${ }^{15} . R n f 168^{-}$mice were generated with the help of the FCCC Transgenic Mouse facility using CRISPR/Cas9 technology. Guide RNA sequences targeting the Rnf168 Ring domain were selected based on high predicted cut efficiency and minimal predicted off-target sites (https://portals. broadinstitute.org/gpp/public/analysis-tools/sgrna-design). Several sgRNAs were tested and ultimately the sgRNA target sequence TCTGTCGTCGCCGGGTCTCT was used. Cas 9 enzyme (IDT, 1081058) and $20 \mathrm{ng} / \mu \mathrm{l}$ sgRNA were resuspended in injection buffer ( $1 \mathrm{mM}$ Tris- $\mathrm{HCl} \mathrm{pH} 7.5,0.1 \mathrm{mM}$ EDTA) and injected into single cell embryos in a B6C3F1/J background (offspring of C57BL/6J females and C3H/ HeJ males, Jax Laboratory). Mice derived from injected embryos were subsequently crossed with C57BL/6 J mice, resulting in a mixed genetic background. Developmental abnormalities in mice such as tail kinks and hypopigmentation were documented after visual inspection. Kaplan-Meier survival was assessed upon mice reaching IACUC guidelines for euthanasia and organs collected, fixed in neutral buffered formalin, paraffin embedded, sections hematoxylin and eosin stained and evaluated by the FCCC Histopathology facility. T-cell lymphoma diagnoses were confirmed by immunohistochemical staining of sections with anti-CD3 antibody (Agilent, M725429-2) and DAB solution development.

Mouse genotyping, embryo analysis, MEF generation, and culture. DNA from mice were extracted with $240 \mu \mathrm{l} 50 \mathrm{mM} \mathrm{NaOH}$ followed by a 10 -min incubation at 95 degrees then neutralized with $60 \mu \mathrm{l} 1 \mathrm{M}$ Tris- $\mathrm{HCl}, \mathrm{pH}$ 8. Genotyping for Brcal ${ }^{C C}$ status was accomplished by PCR amplification using primers (GGTGCACT CTCCTCCAACATC, CTCTTGCACCTGCCTCTCTGA). PCR products were subject to digestion with EcoNI and bands visualized using agarose gel electrophoresis. The $B r c a 1^{C C}$ allele introduces an additional EcoNI cut site, as previously described ${ }^{15}$. Rnf $168^{-}$allele was detected using two PCR reactions with a common primer (GGGTCCTCACCGCGTAAGAAG) and one reaction that amplifies the wild-type gene (CGAAGAGACCCGGCGACGA) and another that amplifies the mutant allele (GTATGGTACCGAGTCCATAAGGGACA) and bands visualized using agarose gel electrophoresis. Pregnant mice were euthanized at 12.5 and 13.5 days post-coitum for assessment of Mendelian developmental ratios and generation of mouse embryonic fibroblast (MEF) cell lines, respectively. Embryos were separated and collected for genotyping. To generate MEF lines, individual embryos were washed with sterile Dulbecco's phosphate-buffered saline (DPBS). Embryos were aspirated 3 times through a $16 \mathrm{G}$ needle and incubated for $15 \mathrm{~min}$ in trypsin/EDTA at 37 degrees. Cells were pelleted by centrifugation for $5 \mathrm{~min}$ at $1000 \times g$, resuspended in $10 \mathrm{ml}$ MEF media (Dulbecco's Modified Eagle's Medium with $4.5 \mathrm{~g} / \mathrm{L}$ glucose, $15 \%$ fetal bovine serum, $1 \times$ GlutaMAX, $1 \times$ MEM nonessential amino acids, $1 \mathrm{mM}$ sodium pyruvate, $1 \times$ penicillin/streptomycin). Prior to passage three, MEFs were immortalized with pBabe-SV40Tag by electroporation using the Amaxa Mouse/Rat Hepatocyte Nucleofector Kit (Lonza, VPL-1004). Immortalized MEF lines were genotyped, tested for mycoplasma and maintained in MEF media with $10 \%$ FBS.

Cell lines. HEK293T were obtained from Takara Bio, MDA-MB-436, MDA-MB231, and MCF7 cell lines were obtained from ATCC, tested for mycoplasma (Lonza, LT07-705) and identities confirmed by short tandem repeat (STR) profiling using IDEXX analysis. Cells were grown at 37 degrees with $5 \% \mathrm{CO}_{2}$ in RPMI or DMEM media with $10 \%$ FBS and $1 \times$ penicillin/streptomycin. 


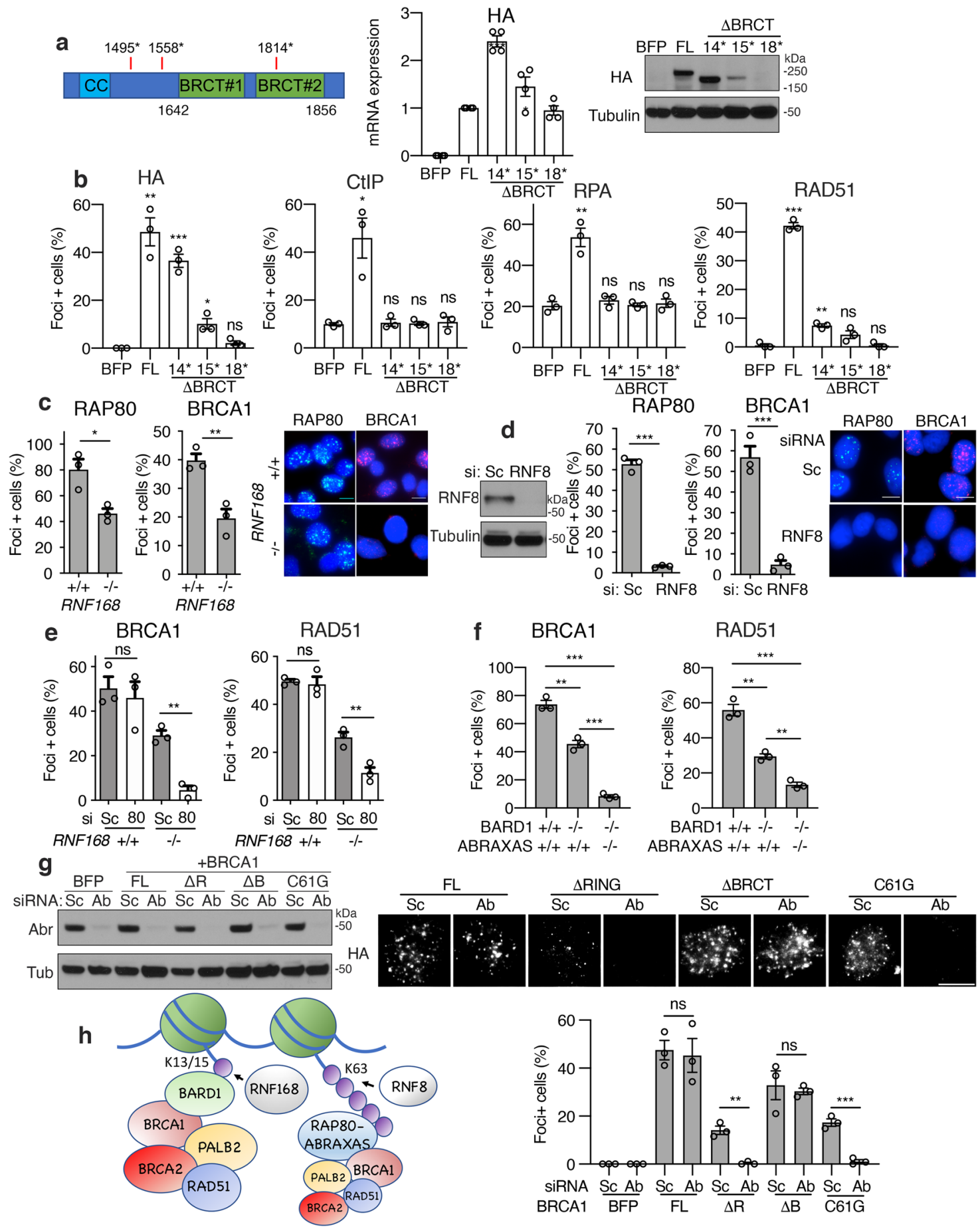

CRISPR/Cas9 edited cell lines. MEF cell lines were transduced with lentivirus containing Cas9 and guide RNA in the lentiCRISPRv2 plasmid (Addgene, 52961) and selected using $2 \mu \mathrm{g} / \mathrm{ml}$ puromycin. Single guide RNA (sgRNA) target sequences were selected based on low predicted off-target score and predicted cutting efficiencies (https://portals.broadinstitute.org/gpp/public/analysis-tools/sgrna-design). Oligos (Supplementary Table 1) were phosphorylated, annealed, then cloned using BsmBI sites. The Rnf168-targeted guide utilized the sequence TCTGTCGTCGCCGGG TCTCT to generate MEF lines. Single cell clones were isolated where indicated and were considered a knockout $(\mathrm{KO})$ if all alleles exhibited frameshifting mutations by Sanger sequencing using the primers described for mouse genotyping. KO was further confirmed by the absence of 53bp1 IRIF. Rap 80 was targeted with ATAGTGA TATCCGATAGCGA sgRNA target sequence and KO confirmed by Sanger sequencing using the following primers; GGTAAAAGGATGCCACGAAGGA and CAAATAATATGACCACACGCGCT. MDA-MB-231 CRISPR/Cas9 edited cell lines were generated by first isolating clones with doxycycline-inducible expression of Cas9 (Addgene, 50661), then infected with lentivirus harboring the sgRNA and selected with $4 \mu \mathrm{g} / \mathrm{ml}$ blasticidin. Surviving cells were subjected to 5 days of $4 \mu \mathrm{g} / \mathrm{ml}$ doxycycline treatment for induction of Cas9 then subclones isolated and screened for KO. RNF168 was targeted with sequences TCGAAAAGGCGAGTTTATGC and ATCTGCATGGAAATCCTCG. Data is shown for a representative clone harboring sgRNA target sequence TCGAAAAGGCGAGTTTATGC. KO was confirmed by Sanger sequencing using primers; TGATACGCTTCTGGGCATAATA and ACCCCCTAACCTCTGAGAACTAT. MCF7 and MDA-MB-436 cells were transduced with lentivirus containing Cas9 and guide RNA in the lentiCRISPRv2 plasmid and selected using $2 \mu \mathrm{g} / \mathrm{ml}$ puromycin. The guide RNA targeting GCGACCATCC GGTTCCATGG was used to knockout BARD1 in MCF7 cells and confirmed by Sanger sequencing using the following primers; GTGCCCTGCGAGTCCCTAT and CAAAAACTACCGTTTCAGTTGGAT. The guide RNA targeting sequence CCTCAACACGGACTCGGACA was used to knockout ABRAXAS in MCF7 cells and confirmed by sequencing with primers; CAGCAGAAGCGAAGGAGGA and GAGGGCTAATGCTGGAGAAGA. The guide RNA targeting GCCATCCAG TCCTCAAGGAG was used to knockout 53BP1 in MDA-MB-436 cells. The expression of 53BP1 in pooled population was confirmed by Western blot using the nuclear extracts. 
Fig. 5 BRCA1 BRCT-ABRAXAS-RAP80 axis is a backup pathway. a Left; cartoon showing BRCA1 BRCT region with aa numbers indicated and positions of stop codon mutations tested. Middle; BRCA1 null MDA-MB-436 cells expressing BFP control, HA-BRCA1-full-length (FL), HA-BRCA1-1495stop ( ${ }^{*}$, HABRCA1-1558*, and HA-BRCA1-1814* were assessed for HA mRNA and divided by a house-keeping gene expression by qRT-PCR, followed by normalization to HA-BRCA1-FL. Mean and S.E.M are shown for $n=4$ technical replicates. Right; cells were assessed for HA protein levels by western blotting. $\mathbf{b}$ Cells from (a) were assessed for HA $(\geq 10)$, CtIP $(\geq 10)$, RPA32 $(\geq 10)$, and RAD51 $(\geq 5)$ IRIF, with the indicated number of foci per nuclei counted as positive and shown as mean and S.E.M. for $n=3$ biological replicates. ${ }^{\star \star \star} p<0.001$, ${ }^{\star \star} p<0.01,{ }^{\star} p<0.05$, ns not significant compared to BFP (unpaired, two-tailed $t$ tests). See Supplementary Fig. 5d for representative images. c MDA-MB-231 RNF168+ $/^{+}$and RNF168 ${ }^{-/-}$cells were examined for RAP80 ( $\left.\geq 10\right)$ and BRCA1 ( $\geq 10)$ IRIF-positive cells, inset representative images, $n=3$ biological replicates. ${ }^{\star \star \star} p<0.001,{ }^{\star \star} p<0.01,{ }^{\star} p<0.05$, ns not significant (unpaired, twotailed $t$-tests). d MDA-MB-231 RNF168 ${ }^{+}+$cells were examined for RAP80 ( $\left.\geq 10\right)$ and BRCA1 ( $\left.\geq 10\right)$ IRIF-positive cells after Sc or RNF8 siRNA and analyzed as described in (c), inset representative images, $n=3$ biological replicates. e Cells from (c) were subject to scrambled (Sc) or RAP80 (80) siRNA treatment and assessed for BRCA1 $(\geq 10)$ and RAD51 $(\geq 5)$ IRIF-positive nuclei and analyzed as described in $(\mathbf{c}), n=3$ biological replicates. See Supplementary Fig. 5e for representative images and RAP80 Western blot. f MCF7 WT, BARD1-/-, and BARD1 ${ }^{-/-}$, ABRAXAS ${ }^{-/-}$DKO cells were examined for BRCA1 and RAD51 IRIF positive cells and analyzed as described in (c), $n=3$ biological replicates. See Supplementary Fig. $5 f$ for representative images. g MDA-MB-436 cells expressing BFP, BRCA1-full-length (FL), BRCA1- $\triangle R I N G(\Delta R)$, BRCA1- $\triangle B R C T(\Delta B)$, or BRCA1-C61G (C61), were subject to Sc or ABRAXAS siRNA and assessed for BRCA1 foci formation. Mean and S.E.M. percentage foci positive cells from three biological replicates are shown. ${ }^{* \star *} p<0.001,{ }^{\star \star} p<0.01,{ }^{\star} p<0.05$, ns not significant (unpaired, two-tailed $t$-tests). h Model for mechanisms of RNF168-mediated BRCA1-P complex recruitment to DSBs. BARD1 BUDR binding to mUb-H2A represents the dominant recruitment pathway, with RAP80-ABRAXAS contributing to a backup pathway.

BARD1, BRCA1, RNF168, PALB2 cloning and cell line generation. Full-length BARD1 (aa 1-777) was first cloned into the pENTR1A Gateway Entry vector (Thermo Fisher Scientific, A10462) with a GFP tag at the N-terminus. The RING domain (aa 34-126), ANK domain (aa 426-521), BRCT domain (aa 560-777), BUDR (R705A, D712A, Q715R) were deleted or mutated using the Q5 SiteDirected Mutagenesis Kit (New England Biolabs, E0554S). The GFP-BARD1 constructs were shuttled into a lentiviral destination vector containing a UBC promoter and a hygromycin resistance gene using the Gateway LR Clonase II Enzyme Mix (Thermo Fisher Scientific, 12538120). BRCA1- $\Delta$ RING ( $\Delta 1-127$ aa, aka M128) and $-\triangle \mathrm{BRCT}\left(\Delta 1496-1863\right.$ aa, aka $\left.1495^{*}\right)$ constructs were generated previously ${ }^{20,29}$. BRCA1-3A (I26A + L63A + K65A), -C61G, - $\triangle$ RING ( $\triangle 1-296$ aa; M297), $-\triangle$ BRCT ( $\triangle 1559-1863$ aa) and $-\triangle B R C T(\Delta 1815-1863$ aa) constructs were generated using site-directed mutagenesis or are described previously ${ }^{20,29}$. BARD1 aa 127-777 was linked using (Gly4Ser)2 to BRCA1 aa 128-1863 to generate a RING deficient BARD1/BRCA1 conjugated protein. The RNF168 cDNA was a gift from Daniel Durocher and was cloned into the pENTR1A Gateway Entry vector with an N-terminal $3 \times$ FLAG tag. RNF168- $\Delta$ RING (aa 2-58) and $-\triangle \mathrm{PID}$ (aa 524-571) domain deletions were generated by PCR and the R57D mutant by the QuickChange Lightning Site-Directed Mutagenesis Kit (Agilent, 210518) according to the manufacturer's instructions. RNF168 constructs were shuttled into identical lentiviral destination vectors containing either a $U B C$ or $C M V$ promoter and a hygromycin resistance gene using the Gateway LR Clonase II Enzyme Mix. Fulllength PALB2 cDNA was cloned into PENTR1A with an N-terminal HA tag, then shuttled into a lentiviral expression vector with a hygromycin resistance gene. BRCA1 RING domain (aa 2-104) was linked using (Gly4Ser)2 to the N-terminal of full-length PALB2 to generate the HA-RING-PALB2 construct. Lentivirus for each construct was produced in HEK293T cells (Takara Bio, 632180) using psPAX2 and VSV-G plasmids. Cell lines were infected with lentivirus using media with $10 \mu \mathrm{g} / \mathrm{ml}$ polybrene (Boston Bioproducts, BM-862W). Cells were then selected using the appropriate antibiotic $(200 \mu \mathrm{g} / \mathrm{ml}$ hygromycin, $2 \mu \mathrm{g} / \mathrm{ml}$ puromycin, or $4 \mu \mathrm{g} / \mathrm{ml}$ blasticidin) or sorted for GFP expression. Construct expression was confirmed by Western blotting.

RNAi experiments. Reverse transfection with Lipofectamine RNAimax (Thermo Fisher Scientific, 13778075) was performed according to the manufacturer's instructions for siRNA experiments. The following siRNA were used: AllStars Negative Control (scrambled) siRNA (Qiagen, SI03650318), pooled RAP80 targeted siRNA (Dharmacon J-006995-09-0002, J-006995-08-0002), pooled RNF168 targeted siRNA (GACACUUUCUCCACAGAUAUU, CAGUCAGUUAAUAGAA GAAAUU, GUGGAACUGUGGACGAUAAUUU), pooled RNF8 targeted siRNA (UGCGGAGUAUGAAUAUGAAUU, GGACAAUUAUGGACAACAAUU, AGA AUGAGCUCCAAUGUAUUU, CAGAGAAGCUUACAGAUGUUU), pooled ABRAXAS targeted siRNA (UAUUAGUGGUAACGUGAUA, CAGGGUACCUU UAGUGGUU).

PARPi colony assays. Cells were seeded in 6 well plates into media containing DMSO or 1-10000 nM of rucaparib. Cells were fixed with 4:1 methanol:acetic acid after 2 weeks, stained with crystal violet, and colonies counted. IC50 calculations were performed with GraphPad Prism software using rucaparib response curves resulting from 3 or more independent experiments. In PARPi resistance assays, cells were seeded at varying densities into DMSO or $50 \mathrm{nM}$ rucaparib and data presented as rucaparib treated colonies normalized to the DMSO treated control colonies for wells where distinct colonies could be counted. Rucaparib was provided by Clovis Oncology.
Immunofluorescence microscopy and foci analyses. Cells were subject $10 \mathrm{~Gy} \gamma$ irradiation (IR) then pre-extracted and fixed at $8 \mathrm{~h}$ post-IR. Pre-extraction was performed on ice for $5 \mathrm{~min}$ with cold cytoskeleton buffer $(10 \mathrm{mmol} / \mathrm{L}$ PIPES $\mathrm{pH}$ 6.8, $100 \mathrm{mmol} / \mathrm{L} \mathrm{NaCl}, 300 \mathrm{mmol} / \mathrm{L}$ sucrose, $3 \mathrm{mmol} / \mathrm{L} \mathrm{MgCl} 2,1 \mathrm{mmol} / \mathrm{L}$ EGTA, $0.5 \%$ Triton X-100) followed by $5 \mathrm{~min}$ with cytoskeleton stripping buffer (10 mmol/L Tris-HCl pH 7.4, $10 \mathrm{mmol} / \mathrm{L} \mathrm{NaCl}, 3 \mathrm{mmol} / \mathrm{L} \mathrm{MgCl}$, $1 \%$ Tween 20 $(\mathrm{v} / \mathrm{v}), 0.5 \%$ sodium deoxycholate). Pre-extracted cells were then fixed at room temperature for $10 \mathrm{~min}$ with $4 \%$ paraformaldehyde, washed with PBS, and treated for 10 min with $1 \%$ Triton X-100 in PBS. Primary antibodies were incubated overnight at 4 degrees in 5\% goat serum in PBS. The following primary antibodies were used: Brcal (gift from A. Nussenzweig; 1:500), BRCA1 (Millipore, 07-434; 1:10,000), RAD51 (Genetex, GTX100469; 1:1000), RAD51 (Abcam, ab133534; 1:50,000), HA (Covance, MMS-101R; 1:1000), BARD1 (Santa Cruz Biotechnology, sc-74559; 1:250), 53BP1 (Novus Biologicals, NB100; 1:1000), 53BP1 (Millipore, MAB3802; 1:10,000), RPA32 (Cell Signaling, 2208; 1:1000), RPA32 (Sigma-Aldrich, NA18; 1:1000), RAP80 (Bethyl Laboratories, A300-763A; 1:10,000), FLAG (SigmaAldrich, F1804; 1:1000), FLAG (Cell Signaling, 14793; 1:1000), CtIP (Millipore, MABE1060; 1:1000). To quantify PALB2 foci, cell lines expressing HA-PALB2 were generated and irradiated cells were stained using the anti-HA antibody. Experiments examining geminin (Abnova, H00051053-M01; 1:15,000) staining did not use preextraction method (to preserve geminin staining). Here, the same protocol was used as above minus the pre-extraction step and beginning at the $4 \%$ paraformaldehyde fixation. Alexa Fluor or FITC conjugated secondary antibodies (Jackson ImmunoResearch Labs or Thermo Fisher Scientific) were incubated for $1 \mathrm{~h}$ at room temperature and slides mounted using Vectashield antifade mounting media with DAPI (Vector Laboratories). Z-stack images were captured using a Nikon NIU Upright Fluorescence microscope and projection images generated using Nikon NIS Elements software. An ImageJ macro was used for quantification of percentage foci positive cells, foci size, and foci per nucleus. Nuclei were identified from the DAPI channel image using the analyze particles function of ImageJ after thresholding the image with the ImageJ median and subtract background functions applied. The identified nuclei were stored as ROI in the ImageJ ROI Manager to allow for characterization of foci within individual nuclei (ROI). Foci identification for each nucleus utilized the ImageJ subtract background, contrast, and gaussian blur functions followed by thresholding and the analyze particles function to generate a foci mask image. Threshold values were manually confirmed and applied consistently within each experiment. Foci per nucleus and foci size quantifications were direct outputs of the analyze particles function (particle count, particle average size) applied to the ROI of each nucleus on the foci mask images and are reported as counts and $\mu \mathrm{m}^{2}$, respectively. Foci positivity was defined as nuclei containing 5 or more RAD51 foci or 10 or more foci for other proteins as indicated in each figure. Percentage foci positive cells are presented as mean and S.E.M. from three independent experiments with the average of each biological replicate shown by open circle data points. Foci per nucleus and foci size data points represent the count and average foci size for individual foci positive nuclei across three independent experiments with the median values indicated. A minimum of four images and 100 nuclei were collected and analyzed per sample in each replicate. For geminin costaining experiments, the ImageJ macro for foci analysis was adapted to include a step for thresholding of the geminin fluorescence image and geminin positivity assessed for individual nuclei.

Western blotting and immunoprecipitation. Nuclear extracts were obtained from pelleted cells with the NE-PER Nuclear and Cytoplasmic Extraction Kit (Thermo Fisher Scientific, 78833) following instructions from the manufacturer and using protease and phosphatase inhibitors (Millipore, 524624, 524625, and 539131). 
Lysates were separated by SDS-PAGE, transferred to a PVDF membrane, and blocked in 5\% nonfat milk in PBST. The primary antibodies were incubated overnight at 4 degrees in $5 \%$ nonfat milk in PBST. The following primary antibodies were used: HA (Cell Signaling, 2367; 1:1000), RNF168 (R\&D Systems, AF7217; 1:1000), RNF168 (Millipore, ABE367; 1:1000), BRCA1 (Millipore, OP92; 1:500), BARD1 (Bethyl Laboratories, A300-263A; 1:1000), GFP (Santa Cruz Biotechnologies, sc-9996; 1:1000), FLAG (Sigma-Aldrich, F1804; 1:1000), V5 (Bethyl Laboratories, A190-120A; 1:1000), PALB2 (Bethyl Laboratories, A301-246A; 1:2000), BRCA2 (Bethyl Laboratories, A303-434A; 1:2000), RAD51 (Santa Cruz Biotechnology, sc-8349; 1:1000), CtIP (Bethyl Laboratories, A300-488A; 1:1000), RAP80 (Bethyl Laboratories, A300-763A; 1:1000), ABRAXAS (Bethyl Laboratories, A302-180A; 1:1000), 53BP1(Millipore, MAB3802; 1:1000), RNF8 (Santa Cruz Biotechnology, sc-271462; 1:1000), Tubulin (Cell Signaling, 2148; 1:2000). HRP conjugated secondary antibodies (Millipore or R\&D Systems) were incubated for $1 \mathrm{~h}$ in 5\% nonfat milk in PBST. V5 tag (Bethyl Laboratories, A190-120A) antibody was used for immunoprecipitation of BRCA1 complexes from $2 \mathrm{mg}$ of nuclear extract using Pierce Classic IP Kit (Thermo Fisher Scientific, 26146) according to manufacturer's instructions.

Cell cycle analyses and RT-PCR. Exponentially growing cells were harvested and fixed with $50 \%$ ethanol at 4 degrees. Cells were washed with PBS and incubated in FxCycle PI/RNase staining solution (Thermo Fisher Scientific, F10797) for $30 \mathrm{~min}$. Data were acquired using a BD FACScan flow cytometer and analyzed using FlowJo software. Total RNA was isolated from cell lines using the RNeasy Plus Mini Kit (Qiagen, 74134). The following primers are located within the $3 \times$ FLAG tag sequence and were used to quantify ectopic FLAG-RNF168 expression: TACAAAGACCATGACGGTGATTA and AGTCTCGCTGCCTGAGATA Quantification of ectopic of BRCA1 FL and - $\triangle$ BRCT expression was performed using primers located on the HA tag and $\mathrm{N}$ terminal of the BRCAl CDNA: CCTACGACGTGCCCGACTA and ATGGGACACTCTAAGATTTTCTGCA. Expression was normalized to $36 \mathrm{~B} 4 \mathrm{mRNA}$ as previously described using q(quantitative) RT-PCR ${ }^{20}$

Statistics and reproducibility. Statistical tests, significant $p$ values, and number of replicates are indicated in the figure legends. Nonparametric Mann-Whitney and unpaired, two-tailed $t$-tests were performed using GraphPad Prism software. R was used for chi-square goodness of fit tests comparing expected and observed genotypes. Each experiment was performed with three biological replicates or as indicated in figure legends.

Reporting summary. Further information on research design is available in the Nature Research Reporting Summary linked to this article.

\section{Data availability}

All relevant data are available from the authors upon reasonable request. The data generated in this study are provided in the Source data file. Guide RNA design was performed using https://portals.broadinstitute.org/gpp/public/analysis-tools/sgrnadesign. Source data are provided with this paper.

Received: 15 June 2021; Accepted: 30 July 2021;

Published online: 18 August 2021

\section{References}

1. Roy, R., Chun, J. \& Powell, S. N. BRCA1 and BRCA2: different roles in a common pathway of genome protection. Nat. Rev. Cancer 12, 68-78 (2012).

2. Huen, M. S., Sy, S. M. \& Chen, J. BRCA1 and its toolbox for the maintenance of genome integrity. Nat. Rev. Mol. Cell Biol. 11, 138-148 (2010).

3. Sy, S. M., Huen, M. S. \& Chen, J. PALB2 is an integral component of the BRCA complex required for homologous recombination repair. Proc. Natl Acad. Sci. USA 106, 7155-7160 (2009).

4. Zhang, F., Fan, Q., Ren, K. \& Andreassen, P. R. PALB2 functionally connects the breast cancer susceptibility proteins BRCA1 and BRCA2. Mol. Cancer Res. 7, 1110-1118 (2009).

5. Zhang, F. et al. PALB2 links BRCA1 and BRCA2 in the DNA-damage response. Curr. Biol. 19, 524-529 (2009).

6. Zhang, F., Bick, G., Park, J. Y. \& Andreassen, P. R. MDC1 and RNF8 function in a pathway that directs BRCA1-dependent localization of PALB2 required for homologous recombination. J. Cell Sci. 125, 6049-6057 (2012).

7. Hu, Y. et al. RAP80-directed tuning of BRCA1 homologous recombination function at ionizing radiation-induced nuclear foci. Genes Dev. 25, 685-700 (2011).

8. Vohhodina, J. et al. RAP80 and BRCA1 PARsylation protect chromosome integrity by preventing retention of BRCA1-B/C complexes in DNA repair foci. Proc. Natl Acad. Sci. USA 117, 2084-2091 (2020).
9. Doil, C. et al. RNF168 binds and amplifies ubiquitin conjugates on damaged chromosomes to allow accumulation of repair proteins. Cell 136, 435-446 (2009).

10. Mattiroli, F. et al. RNF168 ubiquitinates K13-15 on H2A/H2AX to drive DNA damage signaling. Cell 150, 1182-1195 (2012).

11. Stewart, G. S. et al. The RIDDLE syndrome protein mediates a ubiquitindependent signaling cascade at sites of DNA damage. Cell 136, 420-434 (2009).

12. Kim, H., Chen, J. \& Yu, X. Ubiquitin-binding protein RAP80 mediates BRCA1-dependent DNA damage response. Science 316, 1202-1205 (2007).

13. Sobhian, B. et al. RAP80 targets BRCA1 to specific ubiquitin structures at DNA damage sites. Science 316, 1198-1202 (2007). doi:316/5828/1198 [pii].

14. Wang, B. et al. Abraxas and RAP80 form a BRCA1 protein complex required for the DNA damage response. Science 316, 1194-1198 (2007).

15. Nacson, J. et al. BRCA1 mutational complementation induces synthetic viability. Mol. Cell https://doi.org/10.1016/j.molcel.2020.04.006 (2020).

16. Zong, D. et al. BRCA1 haploinsufficiency is masked by RNF168-mediated chromatin ubiquitylation. Mol. Cell 73, 1267-1281.e1267 (2019).

17. Nakamura, K. et al. H4K20me0 recognition by BRCA1-BARD1 directs homologous recombination to sister chromatids. Nat. Cell Biol. 21, 311-318 (2019).

18. $\mathrm{Li}, \mathrm{M} . \& \mathrm{Yu}, \mathrm{X}$. Function of BRCA1 in the DNA damage response is mediated by ADP-ribosylation. Cancer Cell 23, 693-704 (2013).

19. $\mathrm{Wu}, \mathrm{W}$. et al. Interaction of BARD1 and HP1 is required for BRCA1 retention at sites of DNA damage. Cancer Res 75, 1311-1321 (2015).

20. Wang, Y. et al. RING domain-deficient BRCA1 promotes PARP inhibitor and platinum resistance. J. Clin. Invest 126, 3145-3157 (2016).

21. Baer, R. \& Ludwig, T. The BRCA1/BARD1 heterodimer, a tumor suppressor complex with ubiquitin E3 ligase activity. Curr. Opin. Genet Dev. 12, 86-91 (2002).

22. Stewart, M. D. et al. Tuning BRCA1 and BARD1 activity to investigate RING ubiquitin ligase mechanisms. Protein Sci. 26, 475-483 (2017).

23. Brzovic, P. S., Meza, J., King, M. C. \& Klevit, R. E. The cancer-predisposing mutation $\mathrm{C} 61 \mathrm{G}$ disrupts homodimer formation in the NH2-terminal BRCA1 RING finger domain. J. Biol. Chem. 273, 7795-7799 (1998).

24. Joukov, V., Chen, J., Fox, E. A., Green, J. B. \& Livingston, D. M. Functional communication between endogenous BRCA1 and its partner, BARD1, during Xenopus laevis development. Proc. Natl Acad. Sci. USA 98, 12078-12083 (2001).

25. Densham, R. M. et al. Human BRCA1-BARD1 ubiquitin ligase activity counteracts chromatin barriers to DNA resection. Nat. Struct. Mol. Biol. 23, 647-655 (2016).

26. Becker, J. R. et al. BARD1 reads $\mathrm{H} 2 \mathrm{~A}$ lysine 15 ubiquitination to direct homologous recombination. Nature https://doi.org/10.1038/s41586-02103776-w (2021).

27. Luijsterburg, M. S. et al. A PALB2-interacting domain in RNF168 couples homologous recombination to DNA break-induced chromatin ubiquitylation. Elife https://doi.org/10.7554/eLife.20922 (2017).

28. Nacson, J. et al. BRCA1 mutation-specific responses to 53BP1 loss-induced homologous recombination and PARP inhibitor resistance. Cell Rep. 24, 3513-3527.e3517 (2018)

29. Wang, Y. et al. BRCA1 intronic Alu elements drive gene rearrangements and PARP inhibitor resistance. Nat. Commun. 10, 5661 (2019).

30. Thorslund, T. et al. Histone H1 couples initiation and amplification of ubiquitin signalling after DNA damage. Nature 527, 389-393 (2015).

31. Munoz, M. C. et al. Ring finger nuclear factor RNF168 is important for defects in homologous recombination caused by loss of the breast cancer susceptibility factor BRCA1. J. Biol. Chem. 287, 40618-40628 (2012).

32. Wang, B. \& Elledge, S. J. Ubc13/Rnf8 ubiquitin ligases control foci formation of the Rap80/Abraxas/Brca1/Brcc36 complex in response to DNA damage. Proc. Natl Acad. Sci. USA 104, 20759-20763 (2007).

33. Kolas, N. K. et al. Orchestration of the DNA-damage response by the RNF8 ubiquitin ligase. Science 318, 1637-1640 (2007).

34. Mailand, N. et al. RNF8 ubiquitylates histones at DNA double-strand breaks and promotes assembly of repair proteins. Cell 131, 887-900 (2007).

35. Morris, J. R. et al. The SUMO modification pathway is involved in the BRCA1 response to genotoxic stress. Nature 462, 886-890 (2009).

36. Soo Lee, N. et al. TRAIP/RNF206 is required for recruitment of RAP80 to sites of DNA damage. Nat. Commun. 7, 10463 (2016).

37. Guzzo, C. M. et al. RNF4-dependent hybrid SUMO-ubiquitin chains are signals for RAP80 and thereby mediate the recruitment of BRCA1 to sites of DNA damage. Sci. Signal 5, ra88 (2012).

38. Johnson, N. et al. Stabilization of mutant BRCA1 protein confers PARP inhibitor and platinum resistance. Proc. Natl Acad. Sci. USA 110, 17041-17046 (2013).

39. Drost, R. et al. BRCA1185delAG tumors may acquire therapy resistance through expression of RING-less BRCA1. J. Clin. Invest 126, 2903-2918 (2016).

40. Drost, R. et al. BRCA1 RING function is essential for tumor suppression but dispensable for therapy resistance. Cancer Cell 20, 797-809 (2011). 
41. Shakya, R. et al. BRCA1 tumor suppression depends on BRCT phosphoprotein binding, but not its E3 ligase activity. Science 334, 525-528 (2011).

42. $\mathrm{Li}, \mathrm{M}$. et al. 53BP1 ablation rescues genomic instability in mice expressing 'RING-less' BRCA1. EMBO Rep. 17, 1532-1541 (2016).

43. Friedman, L. S. et al. Novel inherited mutations and variable expressivity of BRCA1 alleles, including the founder mutation 185delAG in Ashkenazi Jewish families. Am. J. Hum. Genet. 57, 1284-1297 (1995).

44. Krais, J. J. et al. RNF168-mediated ubiquitin signaling inhibits the viability of BRCA1 null cancers. Cancer Res. https://doi.org/10.1158/0008-5472.CAN-193033 (2020).

45. Callen, E. et al. 53BP1 Enforces distinct pre- and post-resection blocks on homologous recombination. Mol. Cell https://doi.org/10.1016/j.molcel.2019. 09.024 (2019).

46. Cruz-Garcia, A., Lopez-Saavedra, A. \& Huertas, P. BRCA1 accelerates CtIPmediated DNA-end resection. Cell Rep. 9, 451-459 (2014).

\section{Acknowledgements}

This work was supported by US National Institutes of Health (NIH) Grants R01CA214799, R01HL150190, and R01GM135293. J.J.K. was supported by an American Cancer Society-Tri State CEOs Against Cancer Postdoctoral Fellowship, PF-19-097-01DMC, Ovarian Cancer Research Alliance and Phil and Judy Messing grant 597484, and T32 CA009035. We are grateful to Sean Hua and the FCCC Transgenic Mouse facility for help generating mice as well as Genomics, Cell Culture and Cell Sorting facilities. We thank Dr. Ross Chapman for helpful discussions, Dr. Kathy Cai and the FCCC histopathology service for pathological analyses of mouse organs, and Dr. Beth Handorf for guidance with statistical analyses.

\section{Author contributions}

J.J.K., Y.W., P.P., J.B., and A.J.B. designed and performed experiments J.J.K., Y.W., A.J.B., and N.J. analyzed and interpreted data. J.J.K., Y.W. and N.J. wrote the manuscript. N.J. supervised the project.

\section{Competing interests}

The authors declare no competing interests.

\section{Additional information}

Supplementary information The online version contains supplementary material available at https://doi.org/10.1038/s41467-021-25346-4.

Correspondence and requests for materials should be addressed to N.J.

Peer review information Nature Communications thanks the anonymous reviewer(s) for their contribution to the peer review of this work.

Reprints and permission information is available at http://www.nature.com/reprints

Publisher's note Springer Nature remains neutral with regard to jurisdictional claims in published maps and institutional affiliations.

(c) (i) Open Access This article is licensed under a Creative Commons Attribution 4.0 International License, which permits use, sharing, adaptation, distribution and reproduction in any medium or format, as long as you give appropriate credit to the original author(s) and the source, provide a link to the Creative Commons license, and indicate if changes were made. The images or other third party material in this article are included in the article's Creative Commons license, unless indicated otherwise in a credit line to the material. If material is not included in the article's Creative Commons license and your intended use is not permitted by statutory regulation or exceeds the permitted use, you will need to obtain permission directly from the copyright holder. To view a copy of this license, visit http://creativecommons.org/ licenses/by/4.0/.

(C) The Author(s) 2021 